\title{
Revista de Medicina Veterinaria
}

January 2017

\section{Ciencias veterinarias rurales: aportes de las ciencias veterinarias al mundo rural}

\author{
Fernando Nassar Montoya \\ Universidad de La Salle, fnassar@lasalle.edu.co \\ Ernesto Andrés Dalmau Barros \\ Universidad de La Salle, erandalmau@lasalle.edu.co \\ Diego Soler-Tovar \\ Universidad de La Salle, revistamedicinaveterinaria@lasalle.edu.co
}

Follow this and additional works at: https://ciencia.lasalle.edu.co/mv

\section{Citación recomendada}

Nassar Montoya F, Dalmau Barros EA y Soler-Tovar D. Ciencias veterinarias rurales: aportes de las ciencias veterinarias al mundo rural. Rev Med Vet. 2017;(34): 7-8. doi: https://doi.org/10.19052/mv.4265

This Editorial is brought to you for free and open access by the Revistas científicas at Ciencia Unisalle. It has been accepted for inclusion in Revista de Medicina Veterinaria by an authorized editor of Ciencia Unisalle. For more information, please contact ciencia@lasalle.edu.co. 


\section{Editorial}

\section{Ciencias veterinarias rurales: aportes de las ciencias veterinarias al mundo rural}

Es cierto que la institucionalidad, legitimidad y hegemonía de las ciencias veterinarias se evidencian globalmente; pero también que están bajo permanente cuestionamiento, como toda ciencia, sobre su pertinencia en el ambiente cambiante que caracteriza la actualidad. En este contexto paradójico, nos atrevemos a decir que las ciencias veterinarias están hoy en día más vigentes que nunca por los diversos ámbitos en los que pueden y deben contribuir en la salud animal, humana y ecosistémica (en últimas, en la salud pública bajo los conceptos de una salud y de interfaces), entre las cuales se presentan algunas problemáticas críticas concernientes directamente a su área del conocimiento, como las que se evidencian en el mundo rural. No por esto es una coincidencia que instituciones, por ejemplo, la Organización Mundial de Sanidad Animal (OIE) consideren los servicios veterinarios (SV) como bienes públicos y la Organización de las Naciones Unidas para la Alimentación y la Agricultura (FAO) como esenciales en emergencias de enfermedades en animales.

Los territorios rurales han sido un objetivo histórico de las ciencias veterinarias; por lo tanto, la Revista de Medicina Veterinaria, así como el Programa de Medicina Veterinaria y los Posgrados en Ciencias Veterinarias, de la Universidad de La Salle (Colombia), consideraron que era necesario abrir un espacio para dar oportunidad a las disciplinas de mostrar sus contribuciones desde la perspectiva de la ruralidad. Una forma de identificar y resaltar las experiencias aplicadas e investigaciones, de lo cual, se espera, genere la continua reflexión sobre el estado del arte, las necesidades y perspectivas en las diferentes regiones.

El resultado de la convocatoria para la publicación del suplemento de 2017 es más que satisfactorio, si se considera que los trabajos sometidos superaron ampliamente las expectativas que se tenían. Se presentaron trabajos de diversa índole y procedencia geográfica, todos aglutinados bajo un denominador común: pertinencia rural.

Finalmente, este número especial, hace parte de un conjunto de actividades desarrolladas durante 2016 para visibilizar la importancia del trabajo de las ciencias veterinarias y afines en los mundos relacionales de la ruralidad, dentro de las que se destacan: el concurso "Aportes de las ciencias veterinarias lasallistas a la Colombia rural", donde se premiaron los mejores trabajos en tres categorías: docentes-investigadores, egresados y estudiantes; y dos conversatorios, el primero sobre "Articulación de las ciencias veterinarias al sector rural: contribuciones para la paz", y el segundo titulado "Del 
bienestar animal al bienestar humano". Para el Programa de Medicina Veterinaria y los Posgrados en Ciencias Veterinarias de la Universidad de La Salle se consideró pertinente reflexionar en conjunto con egresados, do- centes y estudiantes sobre los significados del desarrollo rural y las responsabilidades de los profesionales de las ciencias veterinarias y afines en el accionar interdisciplinario para contribuir a la paz.

Fernando Nassar Montoya, MV, MSc

Director

Programa de Medicina Veterinaria

fnassar@lasalle.edu.co

Ernesto Andrés Dalmau Barros, MV, MSc

Director

Clínica Veterinaria

erandalmau@lasalle.edu.co

Diego Soler-Tovar, MV, MSc

Editor

Revista de Medicina Veterinaria

revistamedicinaveterinaria@lasalle.edu.co 